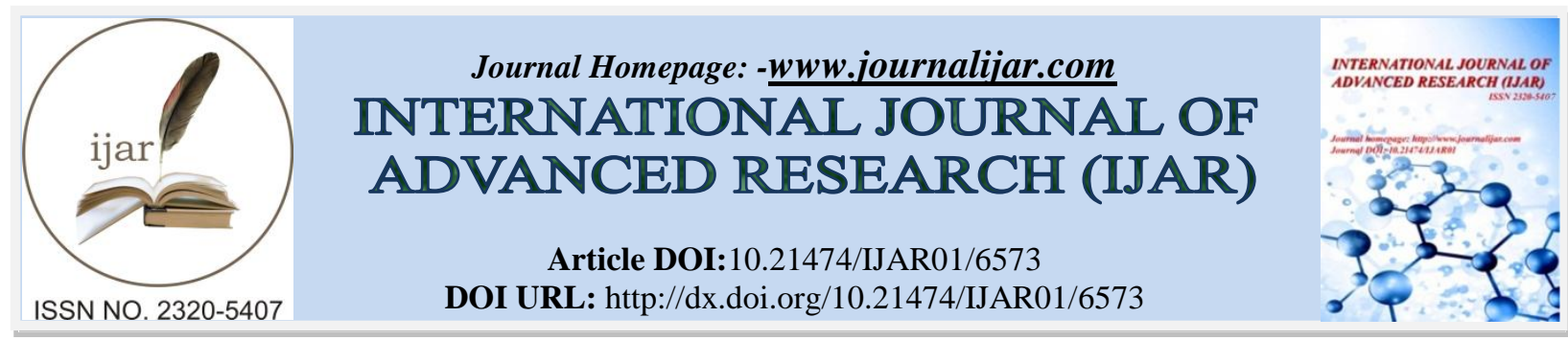

RESEARCH ARTICLE

\title{
LAPAROSCOPIC HYSTERECTOMY UNDER SPINAL ANAESTHESIA.
}

\author{
Dr. Sangeeta Saymote ${ }^{1}$ and Dr. Sushant Gulghane ${ }^{2}$. \\ 1. Assist Professor, Prakash institute of medical sciences, mh, India. \\ 2. Assist prof, Prakash institute of medical sciences, mh, India.
}

\section{Manuscript Info}

Manuscript History

Received: 23 December 2017

Final Accepted: 25 January 2018

Published: February 2018

\section{Abstract}

\section{Introduction:-}

With the advances of laproscopy, hysterectomy is routinely performed laproscopically. It has many advantages as compared to open abdominal hysterectomy such as less post operative pain, better cosmetic results, shorter hospital stay, lower complication rate.Howeverlaproscopic surgeries are routinely performed under general anaesthesia with endotracheal intubation to prevent aspirations of gastric contents and respiratory embarrassment secondary to pneumoperitoneum. But it has several disadvantages like hemodynamic instability, postoperative nausea and vomiting, increased postoperative analgesic requirement, complications related to intubation or extubation and postoperative sore throat.Although there are many reportsoflaproscopic cholecystectomy and laproscopic inguinal hernia repair under regional anaesthesia have been published few studies have involved laproscopic hysterectomy under regional anaesthesia. Use of regional anaesthesia in laproscopic surgery has been limited to patients at high risk for general anaesthesia due to severe coexisting pulmonary, cardiac or ither disease. Regional anaesthesia has been successfully used for laproscopic surgeries unfit for general anaesthesia but not tried in fit patients. Laproscopic surgeries under regional anaesthesia in previous studies is commonly associated with problems such as referred shoulder tip pain secondary to $\mathrm{co} 2$ pneumoperitoneum, patient anxiety, pain, discomfort and inadequate sedation which can be easily managed by intravenous analgesics. Herewith we present case series of 24 patients undergoing total laproscopic hysterectomy under spinal anaesthesia with intrathecal bupivacaine and clonidine..

\section{Methods:-}

After the approval of institutional ethics committee and written informed consent 24 ASA grade 1 and 2 patients in the age group of 35 to 55 years underwent total laproscopic hysterectomy under spinal anaesthesia with intrathecal bupivacaine and clonidine. Exclusion criteria included those with presence of any condition contraindicating elective surgery or spinal anaesthesia.

The patients were explained during pre operative visit about the procedure and also told that any anxiety, pain, or discomfort during the surgery would be dealt with intravenous medications or if required conversion to general anaesthesia.

During and after the procedure patients are encouraged to report any side effects like shoulder tip pain, discomfort, nausea or vomiting. 
All patients are advised overnight fasting and received $0.5 \mathrm{mg}$ alprazolam in the night prior to surgery. In the operative room iv line secured and inj. Odansetron $8 \mathrm{mg}$ and injCeftiaxone $1 \mathrm{gm}$ given intravenously. All patients received $15 \mathrm{ml} / \mathrm{kg}$ RL preloading. All routine monitors ECG, NIBP, Spo2 were attached and baseline vital signs were recorded.

The patients were positioned in sitting position and L2-L3 or L3-L4 space was palpated. Under strict aseptic precautions spinal anaesthesia was given with $26 \mathrm{G}$ quinckes needle and after confirmation of free flow of csf inj. Bupivacaine $0.5 \%$ (heavy) and clonidine $30 \mathrm{mcg}$ given

intrathecally. Patients were turned to supine position and 10 degree trendelenberg tilt was given to achieve required level of block as assessed by pin prick method.

HR, ECG, Spo2 monitors attached and NIBP recorded every 3 minutes for duration of surgery. Once the block was considered adequate (minimum block T4 as assessed by pin prick method) surgery was commenced using co2 insufflation at maximum pressure limit of $12 \mathrm{mmHg}$.

Anxiety if required was treated with iv midazolam $2 \mathrm{mg}$ in divided doses. Shoulder tip pain or any discomfort due to co2 pneumoperitoneum was treated with inj. pentazocine $30 \mathrm{mg}$ in divided doses. Hypotension was treated with fluid bolus or inj. mephentermine $6 \mathrm{mg}$ bolus and $3 \mathrm{mg}$ was repeated as and when required. Bradycardiabolus (HR< 50) was treated with inj. glycopyrolate $0.2 \mathrm{mg}$ iv bolus and if required inj. Atropine $0.6 \mathrm{mg}$ iv.

The surgical procedure of total laproscopic hysterectomy was carried out according to standard protocol with no modification. Operative time as well as any intraoperative event was recored. All the patients were given $100 \mathrm{mg}$ of inj. Tramadol for postoperative pain relief.Inj. Diclofenac sodium $75 \mathrm{mg}$ imsos given in the postoperative period.

\section{Results:-}

24 patients were taken for total laproscopic hysterectomy during the period of dec 2016 to feb 2017. Age group of patients between 40 to 60 years. All the 24 surgeries were completed laproscopically. Average duration for surgery is $90 \mathrm{~min}(60-120 \mathrm{~min})$. None of the patient required conversion to general anaesthesia.

Intraoperative parameters including blood pressure, pulse rate,spo2 and respiratory rate were all within normal limits. 8 patients required pharmacological intervention for hypotension with one time support of injmephentermine $6 \mathrm{mg}$. 6 patients required inj. glycopyrolate $0.2 \mathrm{mg}$ for bradycardia (HR less than 50). Patients were catheterised preoperatively. There was no case of PDPH. All the patients received injpentazocine $30 \mathrm{mg}$ in divided doses for shoulder tip pain.None of the patien developed respiratory depression or required assistance for ventilation.

\section{Discussion:-}

Nowadays laproscopic surgery is becoming gold standard. Previously regional anaesthesia was not considered sutaible for the laproscopic procedures because of the risk of aspiration, discomfort due to co2 pneumoperitoneum, increased load of co2, and shoulder tip pain. General anaesthesia as the only suitable technique for laproscopic procedures is the concept of past as it doesn't facilitate postoperative analgesia and emesis free recovery. Also there are various complications associated with airway instrumentation and risk of unanticipated difficult airway. In addition sympathetic stimulation due to stress response during intubation in GA adds to sympathetic overactivity due to pneumoperitoneum resulting in hypertensive episodes. So in terms of safety and post of morbidity and pain scores regional anaesthesia is a better choice for laproscopic procedures.

This case series provides a indication regarding safety and adequacy of spinal anaesthesia with bupivacaine and clonidine in patients undergoing total laproscopic hysterectomy.

The combination of bupivacaine heavy $0.5 \%$ with clonidine $30 \mathrm{mcg}$ in spinal anaesthesia with 10 degree trendelenberg tilt provides adequate level of sensory blockade a d abdominal relaxation in all our 24 patients undergoing total laproscopic hysterectomy.

None of the patient developed respiratory depression or breathing difficulty as respiratory mechanism remains intact and diaphragm the main respiratory muscle is unaffected allowing the patient to adjust minute ventilation by increasing the respiratory rate. 
Cardiovascular changes were also minimal. Clonidine stimulates Alpha2 adrenergic inhibitory neurons in medullary vasomotor center leading to hypotension and bradycardia but it can easilymanaged with bolus iv fluids and pharmacological intervention. 8 patients required one time support of $6 \mathrm{mg}$ of inj. mephentermine. 6 patients required inj. glycopyrolate for bradycardia $(\mathrm{HR}<50 / \mathrm{min})$.

Shoulder tip pain is quite common during laproscopic procedure under regional anaesthesia due to streching of diaphragm by co2 insufflation as diaphragm is supplied by cervical roots which are spared during regional anaesthesia. In our study all patients received inj. pentazocine 30mg in divided doses once the pneumoperitoneum is induced and maximum pressure limit set up to $12 \mathrm{mmHg}$. Any anxiety due to awareness is treated with inj. midazolam $2 \mathrm{mg}$ iv. Use of Clonidine intrathecally has a sedative effect which is an added advantage in these patients.

Another advantage of regional anaesthesia was reduced incidence of intraop and postop nausea and vomiting (PONV). Adequate hydration, reduced systemic opioid use and pre op prophylaxis by odensetron resulted in reduced incidence. PONV is particularly troublesome after GA due to use of opioids, N2O and reversal agents.

Studies have suggested that intrathecal clonidine prolongs sensory and motor block from spinal anaesthesia and decreases anaesthetic requirement during surgery and increases postoperative analgesia. So there was no restlessness, sore throat, dizziness, relaxant induced muscle pain as commonly seen with GA. Prolonged post operative analgesia resulted in smooth and uneventful recovery of patient.

This study confirms feasibility of spinal anaesthesia as a sole technique in total laproscopic hysterectomy. This study doesnot include cost analysis but other studies indicate laproscopic surgeries under regional anaesthesia are more cost effective than under general anaesthesia. This makes spinal anesthesia with additives like clonidine as an attractive option for total laproscopic hysterectomy.

\section{Conclusion:-}

Total laproscopic hysterectomy has significantly reduced postoperative morbidity and mortality of patients. General anaesthesia as a sole anaesthetic technique for these procedure has negated some of the advantages of these procedures. Regional anaesthesia is much more safer and advantageous to the patients. We did this study to assess feasibility of doing total laproscopic hysterectomy under spinal anaesthesia with additive like clonidine without modification of surgical technique. Our study has confirmed the feasibility of performing these surgeries under spinal anaesthesia as a sole anaesthetic technique. It also provides minimal hemodynamic perturbations, postoperative pain control and patient satisfaction. However this approach requires cooperative patient, experienced surgeon and anaesthetist who is ready to supplement it with intravenous medication if required. Thus we can conclude that if properly applied regional anaesthesia has scope to become gold standard anaesthetic technique for laproscopic procedures in future.

\section{References:-}

1. Col R.K.Singh, Lt.Col A.M. Saini, Lt College NitinHoel, College Dinesh Vishay, College Atul Seth et alMajor laproscopic surgery under regional anaesthesia: A prospective feasibility study Med J Armed Forces(2015) 126-131.

2. Rajesh S Mane, Mhanunach C Patil, K.S. Kedareshwar,C. S Aani kop et al-Combine spinal epidural anaesthesia for laproscopicappendicectomy in adults: A case series Saudi J Anaesthesia 2012;6:27-30.

3. Vretzakis G, Bare ka M, Aretha D, Karnikolas M. Regional anaesthesia for laproscopicsurgery:a narrative review.JAnaest 2014;28(3):429-446.

4. Chui PT, Gin T, oh TE.Anaesthesia for laproscopic general surgery. Anaesth Intensive care.1993;21:163-171.

5. Gerges FJ, Manasi GE, Jabbour- Khoury SI. Anaesthesia for laproscopic:Areview.J Club. Anaesth.2006;18:6778.

6. Arati S, Ashutosh N. Comparative analysis of spinal vs general anaesthesia for laproscopic cholecystectomy: a prospective randomized study.Internet J Anaesthesia.2009;24(1).

7. Agrawal N, Gupta A, Gupta K, Khare S. Feasibility of laproscopic cholecystectomy under spinal anaesthesia. People's J Sci Res.2012;5(2):17-21. 